\title{
Affordable method of video recording for ecologists and citizen-science participants
}

\author{
Eunjeong Yang ${ }^{1}$, Keesan Lee ${ }^{1}$, Jung-moon Ha ${ }^{1}$, Woojoo Kim ${ }^{1}$, Ho-Kyung Song ${ }^{1}$, Injae Hwang ${ }^{1}$, Sang-im Lee ${ }^{1,2^{*}}$ \\ and Piotr G. Jablonski ${ }^{1,3^{*}}$ iD
}

\begin{abstract}
Observations and video documentation of interactions between animals living in dens, cavities, and other enclosed spaces are difficult, but they play an important role in field biology, ecology, and conservation. For example, bird parents visiting nests and feeding their nestlings may provide crucial information for testing of ecological hypotheses and may easily attract attention of participants of citizen-science ecological and conservation projects. Because of the nest concealment of cavity-nesting birds, their behaviors in the nest can only be studied by using video surveillance. Professional wildlife surveillance systems are extremely expensive. Here, we describe an inexpensive video setup that can be constructed with relatively little effort and is more affordable than any previously described system. We anticipate that the relatively low cost of about 250 USD for a battery-operated system is an important feature for citizen-science type of projects and for applications in heavily populated areas where the potential for theft and vandalism may be high. Based on our experiences, we provide methodological advice on practical aspects of using this system in the field for ecological research on birds. We highlight the low cost, easiness of construction, and potential availability to a large number of observers taking part in wildlife monitoring projects, and we offer technical help to participants of such research projects.
\end{abstract}

Keywords: Video surveillance, DVR, Nest box, Method, Nest monitoring, Varied tit, Sittiparus varius

\section{Introduction}

Field biology serves as the ultimate testing ground for the core ideas in evolutionary biology and ecology, yet it is often underfunded and down-played by funding agencies. Yet, it is one of those areas in which many professional enthusiasts or "average citizens" (Silvertown 2009; Sullivan et al. 2009) may contribute importantly to persistent development of knowledge. To facilitate these developments, novel technologies should be made inexpensively and widely available. Video surveillance techniques are one of such areas that contribute to new discoveries in wildlife biology. Particularly, detailed observations of avian parents visiting nests and feeding their nestlings may provide crucial information for testing of evolutionary and ecological hypotheses (e.g., Houston et al. 2013), and nest box breeding birds are models for ecological studies. Because of their nest concealment, avian behaviors in the nest

\footnotetext{
* Correspondence: sangim@dgist.ac.kr; piotrjab@behecolpiotrsangim.org ${ }^{1}$ Laboratory of Behavioral Ecology and Evolution, School of Biological Sciences, Seoul National University, Seoul 08826, South Korea

Full list of author information is available at the end of the article
}

boxes can only be studied by using video surveillance (e.g., Hinde 2006). However, the professional wildlife surveillance systems aiming at large and/or well-funded institutions are not available to many field biologists because they are very expensive. Low cost is a substantial concern for applications in heavily populated areas where the potential for theft and vandalism may be high. Although some less expensive video surveillance alternatives have been already proposed for various purposes in various parts of the world (Sabine et al. 2005; Pierce and Pobprasert 2007; O'Brien and Kinnaird 2008; Cox et al. 2012; Park et al. 2012), they may often be still relatively expensive (e.g., $\sim 700$ USD in Pierce and Pobprasert 2007). Here, we describe a very inexpensive new combination built from widely available components resulting in a battery-operated setup for video recording of bird activity in their nests in field conditions. Based on our experience during one field season, we provide advice on how to efficiently use these setups in the studies on birds that breed in nest boxes.

(c) The Author(s). 2018 Open Access This article is distributed under the terms of the Creative Commons Attribution 4.0 International License (http://creativecommons.org/licenses/by/4.0/), which permits unrestricted use, distribution, and reproduction in any medium, provided you give appropriate credit to the original author(s) and the source, provide a link to the Creative Commons license, and indicate if changes were made. The Creative Commons Public Domain Dedication waiver (http://creativecommons.org/publicdomain/zero/1.0/) applies to the data made available in this article, unless otherwise stated. 


\section{Materials and methods}

\section{Study site and species}

In the breeding season of 2017, we tested 10 video recording systems in a population of the varied tit (Sittiparus varius) breeding in nest boxes on the slopes of Gwanak Mountain, Seoul. The area is heavily used by hikers, especially during weekends.

\section{Description of the video surveillance setup}

Each system (Fig. 1) consisted of five main components (Table 1). We used single Channel Mobile DVR with maximum record capacity of $32 \mathrm{~GB}$ (we used 16- and 32-GB memory cards) that can record date and time. We used CMOS color IR camera, which is sold for exclusive use in nest boxes and focuses from 5 to $55 \mathrm{~cm}$. The timer, LEDs, and the $12 \mathrm{~V} 4.5 \mathrm{AH}$ batteries (Table 1) are easily available in Korea. Because the camera views the nest interior from behind a plastic wall that is IR-opaque (Fig. 1c), we used extra LED lights. We used a series of three LEDs arranged in a row and attached at the nest box's roof (Fig. 1e). The circuit in Fig. 1a allowed to set up the system in the afternoon on the day prior to the day of the morning recording session that started automatically according to timer settings.

Each system was prepared in the lab prior to going to the field. Each camera was attached to a removable side wall of a nest box on the external side of the wall (Fig. 1b, c, i.e., the side that is facing outside of the nest box). We cut a large opening and covered it with a sheet of transparent Plexiglas (on the side of nest box interior; Fig. 1b, c), through which the camera viewed the nest interior. The camera was covered with a plastic box glued to the exterior of the wall (Fig. 1c, d). The plastic box was impenetrable to light. This provided protection from rain and direct sunlight. A small opening at the lower part of the plastic box was made for the camera cables (Fig. 1c, bottom). The DVR, timer, and battery were tightly closed in the second plastic box on the ground (Fig. 1d, f) with all connections hidden in this box for protection against rain. A small hole in the side wall was made for the cables: power cable to camera, power cable to LED, and cable connecting camera with DVR input.

The electric circuit (Fig. 1a) shows that the battery provides power, through a timer, to the DVR, camera, and LED. The connection to DVR is short and entirely within the plastic box (Fig. 1e). The connections to camera and LED are through a long cable that runs from the plastic box located at the bottom of the tree upwards to the nest box (Fig. 1d) with camera and LED installed. The connections between cables are within plastic boxes (on the ground or on the side of the nest box).

\section{Deployment in the field}

The procedure of setting up the system in the field takes about 30-40 min. First, the original side wall is removed and replaced by the wall with the camera box (Fig. 2a). Then, the nest cup is shifted toward the camera holding wall such that the opposite side of the nest comprises a platform on which the parents are likely to stand when they feed nestlings (Fig. 2b; Fig. 3a, b). Then, the LED

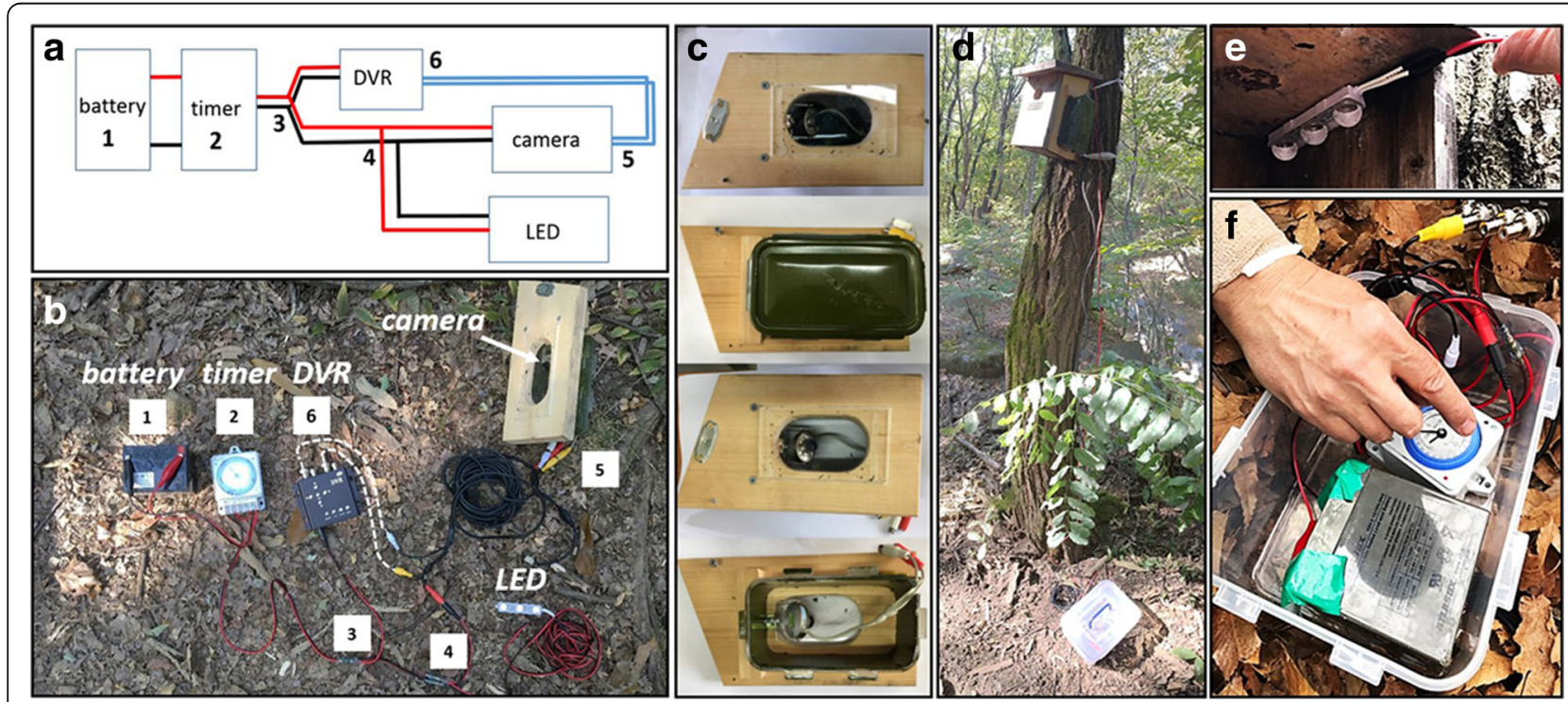

Fig. 1 Video-recording setup and its application in the field. Electric circuit (a) and the actual system (b) set on the forest ground before application with crucial elements labeled and crucial connections numbered. c The upper part the wall with the camera setup closed in the opaque plastic box is shown, and the corresponding view after opening the lid of the opaque box is shown in the bottom part of $\mathbf{c}$. $\mathbf{d}$ View of a nest box with the camera system ready to record. e Location of LED under the roof of the nest box using double-sided sticky tape. $\mathbf{f}$ The box containing the battery, timer, and DVR during the process of setting up the system in the field 
Table 1 List of the main components for one camera-DVR setup and their approximate cost in USD

\begin{tabular}{|c|c|c|c|c|}
\hline Component & Approximate costs (USD) & Model & Manufacturer & $\begin{array}{l}\text { Dimensions: width } \times \\
\text { length } \times \text { height }(\mathrm{mm})\end{array}$ \\
\hline DVR machine & 100 & $\begin{array}{l}\text { High Definition Single Channel Mobile } \\
\text { DVR (MDVR25HR) }\end{array}$ & China & $120 \times 100 \times 30$ \\
\hline Camera & $\begin{array}{l}40 \text { ( } 80+\text { for better camera) single } \\
\text { camera or camera kit with cables } \\
\text { etc. }\end{array}$ & $\begin{array}{l}\text { Removable CMOS color IR camera with } \\
\text { microphone and phono connectors } \\
\text { (HK100137) }\end{array}$ & $\begin{array}{l}\text { Handykam, } \\
\text { Cornwall, UK }\end{array}$ & $35 \times 40 \times 30$ \\
\hline Timer & 50 & Analogue timer (HTS-24B) & $\begin{array}{l}\text { HAN_SEUNG } \\
\text { AUTOMATIC, } \\
\text { Korea }\end{array}$ & $77.5 \times 112.5 \times 42.3$ \\
\hline Battery & 40 & $12 \mathrm{~V} 4.5 \mathrm{AH}$ battery & & $90 \times 70 \times 101(1.6 K G)$ \\
\hline $\begin{array}{l}\text { LED, plastic box, power } \\
\text { cables, connectors, etc. }\end{array}$ & 20 & $\begin{array}{l}12 \vee 3 \text { bulbs LED light (IR LED can be } \\
\text { used too) }\end{array}$ & LEDCT, Korea & $15 \times 60 \times 17$ \\
\hline Total & $\begin{array}{l}250 \text { ( } 290 \text { or more for kit with better } \\
\text { camera) }\end{array}$ & & & \\
\hline
\end{tabular}

lights are installed on the roof using a double-sided sticky tape (Fig. 1e, Fig. 2c) and the LED cable is inserted in a space between the side wall and the roof. The side wall must be properly prepared to provide such a space (hole) for the LED cable. Finally, the cables are connected in the plastic box on the ground, the camera angle is adjusted while watching the image on a portable monitor temporarily connected to the output from the DVR, the timer is set, and the plastic box is closed. The system is then ready for recording (Fig. 2d) and the portable monitor is disconnected, plastic box on the ground tightly closed and covered with vegetation to provide some degree of camouflage.

We have setup the system at 23 nest boxes to record 3 days in a row, each day from 7 am to 9:30 am during the time when nestlings were young $(2,3$, and 4 days old) and later when they were older (7, 8, and 9 days old). We setup the system either during the second week of incubation or a day or two before the first day of filming. One day before the first morning of recording, we
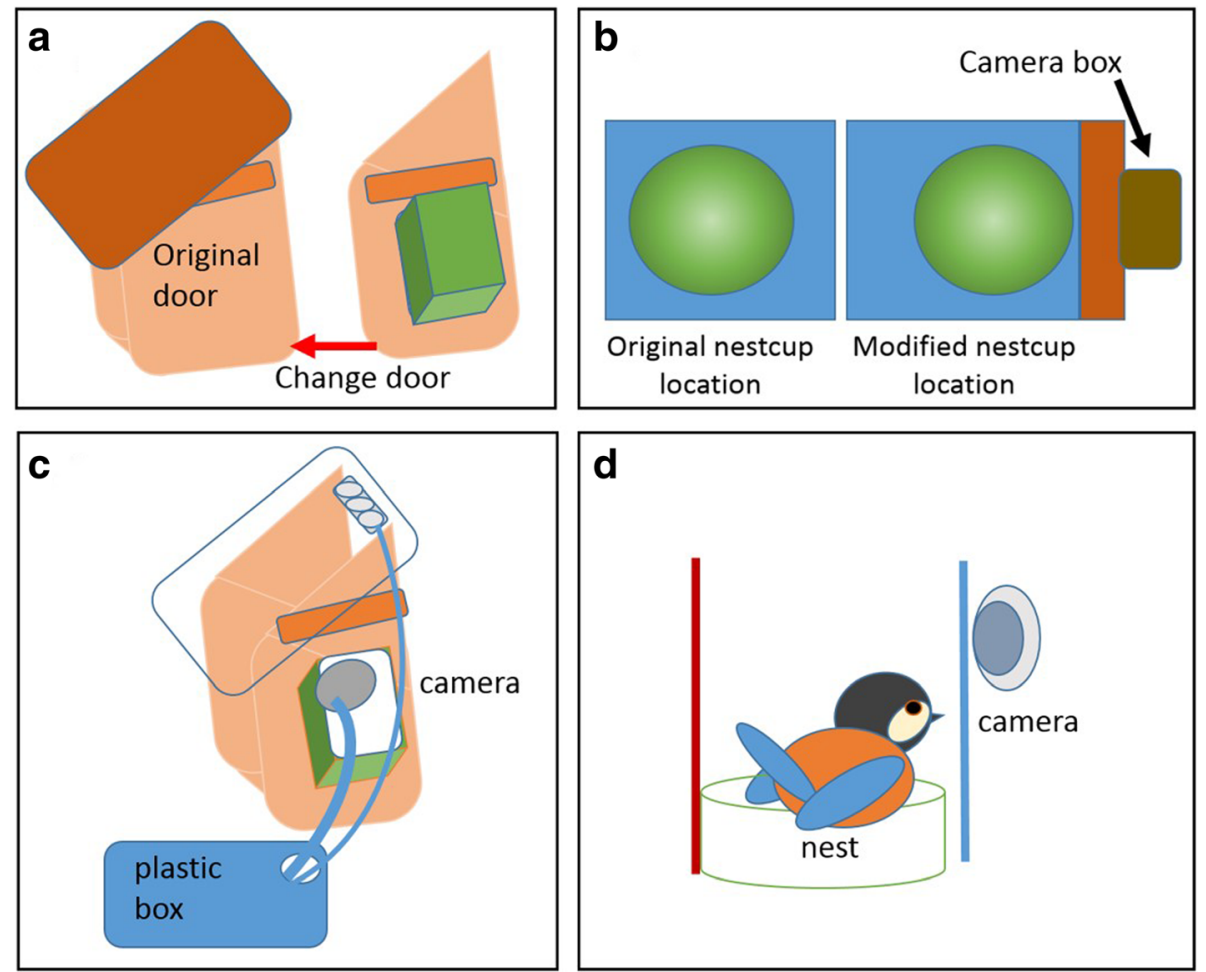

Fig. 2 Schematics of setting up in the field. a Replacing the original wall with camera-holding wall. $\mathbf{b}$ Rearranging the nest material to shift the cup closer to the camera wall. c Connecting the camera to the DVR, timer, and battery. $\mathbf{d}$ The setup ready for recording 


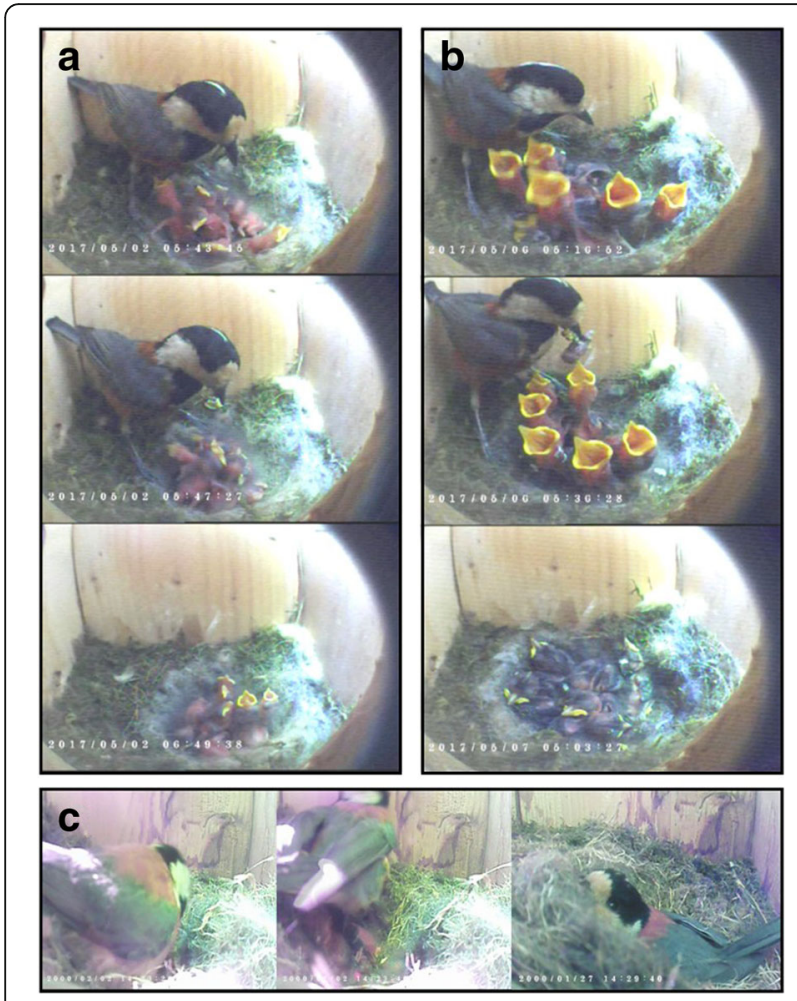

Fig. 3 Examples of frames from good movies recorded at the early stage (a 2-4 days old nestlings) and at the older stage (b 7-9 days old nestlings). c Examples of frames from "bad" movies

carefully approached the nest tree, opened the box on the ground, connected the battery, set the timer, checked the view provided by the camera using a portable monitor connected to the DVR video outputs, disconnected the monitor, closed the box, and left the area.

\section{Results and discussion}

\section{Examples of good recordings}

We obtained good movies if the camera angle and focus were set properly and if the nest cup was shifted towards the camera providing a platform on which the parents stood while feeding nestlings (Fig. 3a, b). In the movie clips, it is possible to identify the feeding parent from the color bands on their legs. The begging behavior of nestlings can also be quantified.

\section{Lessons for future applications}

We monitored 23 nests and planned to obtain 46 sets of 3 -day recordings (each day $2.5 \mathrm{~h}$ ). We recorded a total of 23 technical failure events (Table 2), of which 10 were directly caused by our mistakes (such like not fully charged battery and insufficient memory on a card). They can be easily avoided in the future. For example, by using a battery of higher capacity (12 V 18AH), we could safeguard against the issue of a not fully charged battery. Technical issues with bad connections (nine times) will also be
Table 2 Causes of technical failures of the equipment

\begin{tabular}{ll}
\hline Description of the technical problem & No. of nests affected \\
\hline Connection problems & 9 \\
Memory card shortage & 6 \\
Disturbance by hikers/humans & 4 \\
Battery not fully charged & 4 \\
\hline
\end{tabular}

avoided after we change the connection method by either directly soldering the two cables or using two pin harness method. At four (out of total 23) nest boxes, the hikers created a disturbance by opening the nest box (at three nest boxes) and dis-attaching the cables (at one nest box). No theft occurred but this may be specific to Korea, and in other locations, stealing of the equipment has to be counted into the costs. The disturbance by hikers can be partially avoided by making sure that the side wall of the box cannot be opened without a screwdriver. Finally, two nests failed due to predation and two were abandoned at incubation stage. Abandoning was not directly related to installing the camera setup but to installing it soon after seriously disturbing the incubating female and not giving her sufficient time before sunset to cease being nervous and to enter the nest again.

We believe that these negative effects can be avoided (1) by letting the female leave the nest naturally rather than being scared by opening the nest box, (2) by not disturbing her otherwise during the day when camera setup is it to be installed, and (3) by installing the camera setup before noon and in the absence of the birds.

Some of the movies were not good for extracting scientific data (Fig. 3c), and this was usually caused by wrong camera angle or by the bird located too close to the camera such that the view was blocked by the parent (e.g., Fig. 3c, left panel). In this situation, the nestlings were not visible as well as the parent's color bands were not fully visible. This was largely related to how the nest cup is located inside the nest box. For good recordings, the researcher should shift slightly the nest cup location towards the camera, creating a larger platform on the side opposite to the camera (Fig. 2b). The parents tend to use this platform and in such cases, their bands are visible and their feeding to each individual nestling can be recorded.

The nest material should also be properly re-arranged every time when the wall with camera is attached to make sure that there is no space between the nest and the new side wall (with camera), because young nestlings may fall into this space and die out of hunger and exposure to elements.

\section{Conclusions}

In summary, after applying the lessons from the first season, we are confident that this inexpensive and 
easy-to-build system provides an efficient way to collect scientific data on parent-offspring interactions, parental care, and other aspects of breeding biology of birds in nest boxes by professionals and amateurs. Each setup is built with commercially available elements, and the cost of about 250-290 USD is significantly below the costs of previously described systems for similar tasks (e.g., reviewed by Cox et al. 2012). We offer help in building these video systems. For technical advice, you can contact the co-author who built the system, Jung-moon $\mathrm{Ha}$, at half_wec@naver.com.

\section{Abbreviations}

CMOS: Complementary metal-oxide-semiconductor; DVR: Digital video recorder; IR: Infrared light; LED: Light-emitting diode; USD: United States dollars

\section{Acknowledgements}

We thank the members of the Laboratory of Behavioral Ecology and Evolution for help. We thank the local Gwanak-gu district for providing us with permits to study Paridae in nest boxes in Gwanak Mountain.

\section{Funding}

Funding was provided from the private funds of the participants and from the BK 21 program and partly from NRF grants 2016R1D1A1B03934340 and 2013R1A2A2A01006394.

\section{Availability of data and materials}

The datasets during and/or analyzed during the current study are available from the corresponding author on reasonable request.

\section{Authors' contributions}

JMH designed and built the systems. All authors contributed to the field work. EY transcribed the videos and wrote the first draft. PGJ and SIL wrote the final text. All authors contributed to shaping of the paper. All authors read and approved the final manuscript.

\section{Ethics approval}

The experimental procedure was approved by the Institutional Animal Care and Use Committee of Seoul National University, SNU-180129-5.

\section{Competing interests}

The authors declare that they have no competing interests.

\section{Publisher's Note}

Springer Nature remains neutral with regard to jurisdictional claims in published maps and institutional affiliations.

\section{Author details}

'Laboratory of Behavioral Ecology and Evolution, School of Biological

Sciences, Seoul National University, Seoul 08826, South Korea.

${ }^{2}$ Daegu-Gyeongbuk Institute of Science and Technology School of

Undergraduate Studies, Daegu 42988, South Korea. ${ }^{3}$ Museum and Institute of Zoology, Polish Academy of Sciences, Wilcza 64, 00-679 Warsaw, Poland.

Received: 27 February 2018 Accepted: 28 May 2018

Published online: 26 June 2018

\section{References}

Cox, W. A., Pruett, M. S., Benson, T. J., Chavacci, S. J., \& Thompson III, F. R. (2012). Development of camera technology for monitoring nests. In C. A. Ribic et al. (Eds.), Video surveillance of nesting birds. Studies in avian biology (no. 43) (pp. 185-210). Berkeley: University of California Press.

Hinde, C. A. (2006). Negotiation over offspring care? - a positive response to partner-provisioning rate in great tits. Behavioral Ecology, 17, 6-12.
Houston, A. I., Szekely, T., \& McNamara, J. M. (2013). The parental investment models of Maynard Smith: a retrospective and prospective view. Animal Behavior, 86, 667-674.

O'Brien, T. G., \& Kinnaird, M. F. (2008). A picture is worth a thousand words: the application of camera trapping to the study of birds. Bird Conservation International, 18, S144-S162.

Park, C. R., Yang, H. M., Oh, J. H., \& Kang, I. (2012). Development of "nest finder system" and its application in forest ecosystems. Korean Journal of Nature Conservation, 6, 27-32.

Pierce, A. J., \& Pobprasert, K. (2007). A portable system for continuous monitoring of bird nests using digital video recorders. Journal of Field Ornithology, 78, 322-328.

Sabine, J. B., Meyers, J. M., \& Schweitzer, S. H. (2005). A simple, inexpensive video camera setup for the study of avian nest activity. Journal of Field Ornithology, 76, 293-297.

Silvertown, J. (2009). A new dawn for citizen science. Trends in Ecology and Evolution, $24,467-471$.

Sullivan, B. L., Wood, C. L., Marshall, J. I., Bonney, R. E., Fink, D., \& Kelling, S. (2009). eBird: a citizen-based bird observation network in the biological sciences. Biological Conservation, 142, 2282-2292.
Ready to submit your research? Choose BMC and benefit from:

- fast, convenient online submission

- thorough peer review by experienced researchers in your field

- rapid publication on acceptance

- support for research data, including large and complex data types

- gold Open Access which fosters wider collaboration and increased citations

- maximum visibility for your research: over $100 \mathrm{M}$ website views per year

At BMC, research is always in progress.

Learn more biomedcentral.com/submissions 